\title{
Escultura y entorno urbano: la consolidación del mito en la ciudad de Valencia
}

Sculpture and urban environment: the consolidation of myth in the city of Valencia

Noelia Gil Sabio

Escuela de Doctorado. Universidad Católica de Valencia San Vicente Mártir, España (noelia.gil@ucv.es)

RESUMEN: El presente artículo se propone como objetivo principal el mostrar el vínculo entre la escultura pública y su entorno urbano y arquitectónico. Y cómo a través de ellas se establece y define el imaginario colectivo de los ciudadanos. Mediante el estudio de cuatro esculturas sitas en diferentes emplazamientos de la ciudad de Valencia, se llevará a cabo una lectura de la obra en su conjunto, incluyendo el contexto urbano que la acoge.

PALABRAS CLAVE: escultura pública, Valencia, urbanismo, mito, entorno urbano

\begin{abstract}
The main objective of this article is to show the link between public sculpture and its urban and architectural environment. And how through them the collective imaginary of citizens is established and defined. Through the study of four sculptures located in different locations in the city of Valencia, a reading of the work as a whole will be carried out, including the urban context that houses it.
\end{abstract}

KEYWORDS: public sculpture, Valencia, urbanism, myth, urban environment 


\section{Introducción}

Este artículo se enmarca dentro del proyecto de investigación PRUCV 20115624 - PN Los mitos fundacionales y sus registros de la Universidad Católica San Vicente Mártir de Valencia anualidades 2015-2017, el cual pretende destacar el vínculo entre escultura urbana y el emplazamiento público que la acoge.

En la representación visual de los mitos, el arte se revela como un elemento capaz de hacer visible y actual el mismo relato histórico. De hecho, en esta faceta misma de "dar vida" al mito, el arte conforma un imaginario colectivo a través del cual los ciudadanos establecen y ratifican su propia identidad. Ángeles custodios, patronos locales y personajes locales son encumbrados al esculpirse en piedra y levantarse a los ojos de los demás como fórmula de cohesión social (Cardells, 2012, pp. 1137-1148).

De esta forma, el triángulo escultura-urbanismo-mito no es algo contemporáneo, ya en el pasado se sirvieron de la colocación de obras escultóricas en lugares concretos de importantes ciudades europeas con la intención de conmemorar la figura o gesta de cierto gobernante o rey. Sin embargo, será durante el siglo XIX, durante el auge de las reformas en las más importantes ciudades europeas, cuando los monumentos conmemorativos sean tratados a modo de "publicidad institucional" (Lecea, 2006, p. 14):

"se convierte en instrumento de propaganda de los nuevos regímenes, de sus héroes y de sus hazañas y al mismo tiempo en elemento de caracterización y de singularización de un espacio público determinado. Los monumentos aparecen como hitos de referencia, urbana que al tiempo que consolidan y completan la tercera dimensión de un espacio urbano que es esencialmente plano, le añaden una dimensión temporal: el tiempo de la Memoria."

A través de la selección de diversas obras escultóricas, teniendo en cuenta la estrecha relación entre los personajes que representan y el valor que tienen para la historia de la ciudad de Valencia, se analiza la íntima conexión y vínculo, en muchos casos ignorada por mismos los ciudadanos, y que ofrece como resultado un conjunto armonioso en continuo diálogo y revisión histórica del mito. Las esculturas públicas seleccionadas para sostener nuestra argumentación son:

El prócer Marqués de Campo, los santos patronos de la ciudad San Vicente Ferrer y San Vicente Mártir, y el jurado Francesc de Vinatea. 


\section{Escultura y entorno urbano}

Los elementos artísticos decorativos de las ciudades - esculturas y monumentos conmemorativos-se entremezclan en el trazado urbano creando y actualizando una red que une el urbanismo y la historia con el ciudadano. Un entramado que en la mayoría de los casos es invisible, haciendo que la historia local sea desconocida.

Mediante cuatro esculturas seleccionadas de la ciudad de Valencia, se pretende establecer y exponer la existente y real vinculación que existe entre esas obras artísticas y su emplazamiento en la ciudad Valencia ${ }^{1}$ :

- El monumento al Marqués de Campo [1]: monumento conmemorativo promovido por el Ayuntamiento de Valencia, situado en la plaza Cánovas del Castillo (Gran Vía Marqués del Turia)y realizado por Mariano Benlliure Gil hacia $1908^{2}$. Su ubicación original era la plaza en el llamado Huerto de Pelaires, frente al Asilo de Párvulos, que llevaba el nombre del prócer, en la calle Beneficencia (actual c/ Corona).

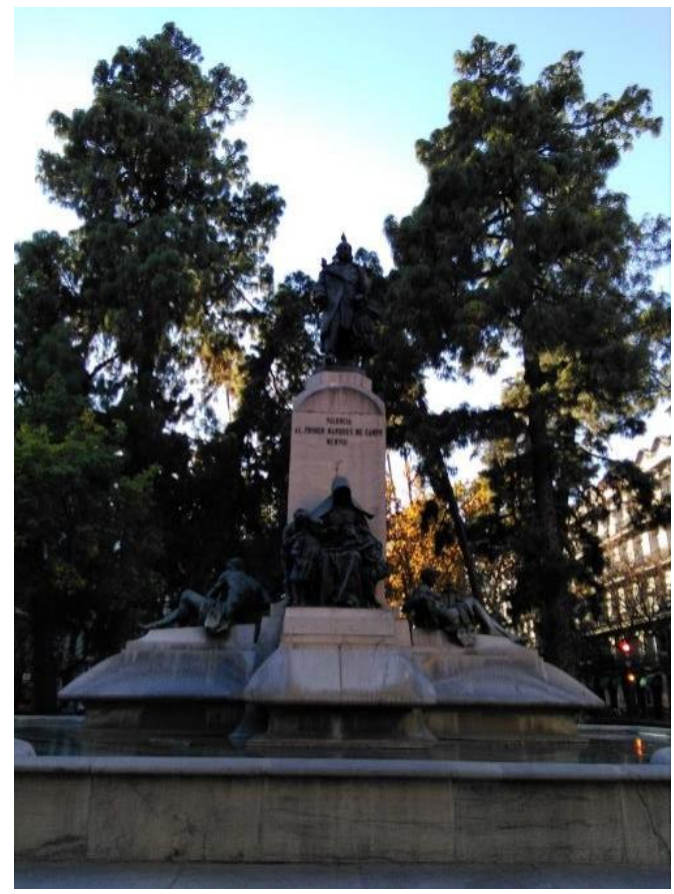

1. Monumento conmemorativo al Marqués de Campo

1 Para la descripción de las obras se han trabajado dos textos: DE LAS HERAS ESTEBAN, Elena (2003), La escultura pública en Valencia. Estudio y catálogo. Universitat de València, Servei de publicacions: Valencia; GIL SALINAS, Rafael (2003), "La escultura pública en Valencia y los artistas valencianos", en LACARRA DUCAY, Carmen y GIMÉNEZ NAVARRO, Cristina (ccord.), Historia y política a través de la escultura pública 1820-1920. Institución Fernando el Católico: Zaragoza, pp. 63-88 y MONTOLIU SOLER, Violeta (2002), Monumentos conmemorativos de Valencia, memoria esculpida de una ciudad: 1875-1936. Real Academia de Cultura Valenciana: Valencia y la Revista de escultura pública Escultura urbana. Recuperado de http://www.esculturaurbana.com (Consulta: 17/11/2017).

2 Sobre la fecha de su realización no existe un acuerdo, habiendo diversidad de opiniones entre los investigadores. Las fechas oscilan entre 1885 y 1911. 
El monumento, con estructura en piedra, está coronado por una escultura en bronce del Marqués de Campo a cuyos pies se colocaron diversas alegorías, igualmente en bronce, -el Ferrocarril, la Marina, el Gas, y la Caridad-y que en su conjunto hacen referencia tanto a la labor emprendedora como a las reformas que promovió en diversos ámbitos de la ciudad y sociedad valencianas (Martínez Roda, 1998):

- Implantó la primera línea de ferrocarril de la ciudad: Valencia-Xàtiva; posteriormente conectó otras localidades cercanas, para finalmente hacerlo con Almansa, Tarragona y Barcelona.

- Reformó diversas instalaciones del puerto marítimo.

- La nueva iluminación pública de la ciudad de Valencia con luz de gas la realizó la empresa del marqués en 1844, partiendo del alumbrado en el jardín de la Glorieta

- De entre los numerosos ejemplos del marqués destacar la construcción de un Asilo de párvulos, que llevaba su nombre, y estaba gestionado por las Hermanas de la Caridad.

La escultura se encuentra en el denominado Eixample, ensanche, de la ciudad de Valencia $(1887)^{3}$, proyecto urbanístico del XIX que fue proyectado por los arquitectos valencianos José Calvo, Luis Ferreres y Joaquín María Arnau, tomando como modelo el Plan Cerdà de Barcelona (1858); fue desarrollado en un ambiente artístico, cultural y económico marcado por la modernidad haciendo que la ciudad de Valencia creciera y se desarrollase al igual que habían hecho, o estaban haciendo, otras capitales de la península (Mestre, 2011, p. 90):

"La Valencia de la segunda mitad del siglo XIX se encuentra cargada de expectativas y novedades: se acaban de derribar las murallas (1865) y la ciudad vislumbra posibilidades de expansión y comunicación: en 1876 se inaugura la primera línea de tranvía, en 1892, el primer tranvía a vapor. La industria de la cerámica vive un gran auge a finales de siglo, se desarrollan los servicios de distribución de alumbrado eléctrico (1886-1889) y teléfono. [...] Entre 1888 y 1917, se construyen cinco líneas de ferrocarril económicas o de vía estrecha en la provincia de Valencia, que posibilitan la comunicación de la capital con sus comarcas limítrofes".

El llamado Ensanche noble -zona extramuros de la ciudad- entre la c/ Colón y la Gran Vía Marqués del Turia, es sinónimo de arquitectura modernista. En sus calles rectas y anchas arquitectos como Demetrio Ribes, Francisco Mora, Manuel Peris o Francisco Almenar, entre otros,proyectaron edificios de gran calidad arquitectónica y estilística que se enmarcan dentro de las tendencias artísticas de principios de siglo: art nouveau, neogótico, eclepticismo y art decó.

3 La tipología del ensanche de Valencia se caracteriza por manzanas cuadradas, de unos 100 metros, con vértices achaflanados. 
- Sant Vicent Ferrer [2]: monumento conmemorativo en mármol y arenisca que representa a uno de los santos patronos de la ciudad de Valencia, junto a San Vicente Mártir, y realizado por José Cloostermans y Vox en 1835. San Vicent Ferrer, fundador, taumaturgo, asesor de Papas y Reyes, decisivo en el Compromiso Sucesor celebrado en la villa de Caspe, es el religioso valenciano más universal (Forcada y Garganta, 1956).

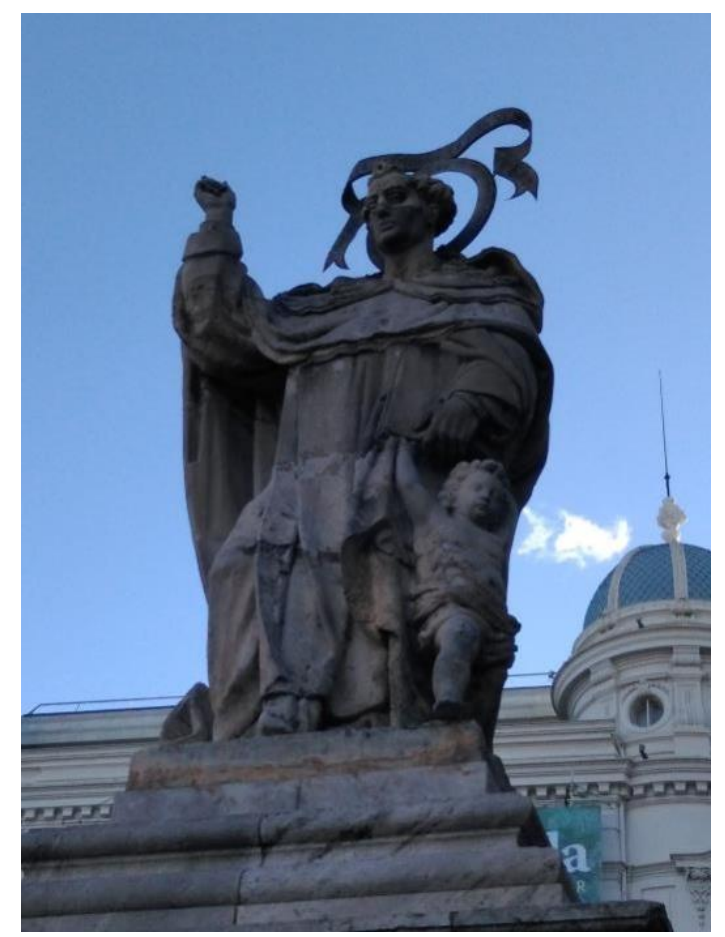

2. Escultura de San Vicente Ferrer

El escultor ha representado al santo patrono vicentino vestido con el hábito dominico, el dedo índice alzado hacia el cielo ${ }^{4}$, un libro en su mano izquierda -el Evangelio-, un ángel y su cabeza rodeada por una filatelia con una frase a la que recurría con asiduidad en sus sermones ${ }^{5}$.

La escultura está situada desde 1960 en la plaza de Tetuán, frente al Convento de Santo Domingo O. P. La importancia del convento, en el que vivió y fue prior el santo valenciano, se remonta a su origen, el cual está ligado al rey Jaume I el Conquistador y a la misma recuperación de la ciudad en $1239^{6}$. Su desarrollo en los siglos posteriores dio como resultado no sólo mejoras en su arquitectura y elementos artísticos - capilla de los Reyes, gran capilla del Rosario, refectorio, claustro

4 El dedo levantado hacia el cielo hace referencia al milagro del albañil, así como indicar que es del cielo de donde proceden todas las gracias y no del santo.

5 "Timete Deum et date illi honorem quia venit hora iudicius eius..." (Ap. 14, 7).

6 Ubicado a orillas del río Turia, el convento de dominicos surge por la donación del soberano Jaume I a su confesor el fraile Miguel de Fabra de unos solares entre las puertas del Real y del Mar. 
mayor...-- ${ }^{7}$, sido por haber sido sede de las Cortes Valencianas. Tras la invasión francesa se convirtió en cuartel y aunque volvió a tener durante un breve periodo de tiempo entre sus muros a una comunidad de frailes dominicos, la desamortización eclesiástica de los liberales condujo a su exclaustración en 1839 pasando a depender del Ministerio y convertirse en sede de la Capitanía General $^{8}$.

- San Vicente Mártir [3]: monumento de carácter conmemorativo y realizado en mármol por el escultor José Cloostermans y Vox en 1835, desde 1960 se encuentra situado en la Gran Vía de Ramón y Cajal, frente a San Vicente de la Roqueta. El escultor ha representado al santo con la rueda de molino, las aspas -haciendo referencia a los elementos de su martirio-, y la dalmática -que representa su condición de diácono- sobre alba talar. En su rostro el escultor ha sabido plasmar la intensidad dramática del personaje que pasa por ser el ejemplo del martirologio más significativo de Europa a lo largo de toda la Edad Media gracias a la difusión a partir de san Agustín de Hipona en el siglo V (Navarro, 2016, pp. 131-146). San Vicente es una de las figuras eclesiales con mayor relevancia para la historia de la ciudad de Valencia, junto a San Vicente Ferrer.

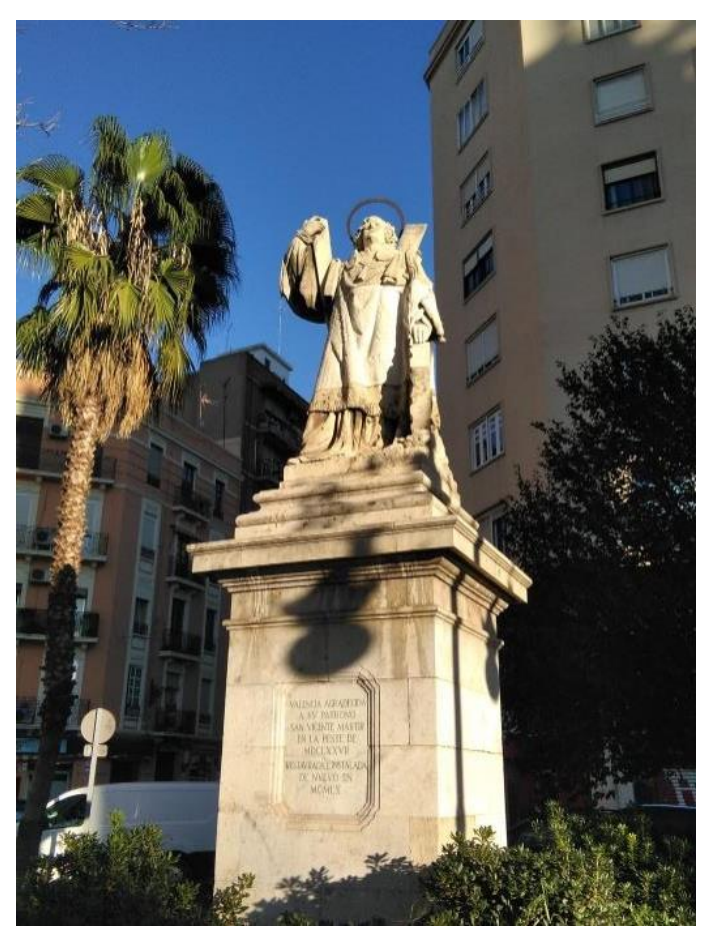

3. Escultura de San Vicente Mártir

7 APARICI NAVARRO, Miguel (2005). Guía del Convento de Santo Domingo, Antigua Capitanía General y sede actual de la Fuerza de Maniobra. Bancaja: Valencia.

8 ZARAGOZÁ CATALÁN, Arturo (1995), "Antiguo Convento de Santo Domingo" Direcció General de Patrimoni Artístic: Valencia en ZARAGOZÁ CATALÁN, Arturo (1995). Valencia, Arquitectura Religiosa. Tomo X, en (1995). Monumentos de la Comunidad Valenciana. Direcció General de Patrimoni Artístic: Valencia 
Con la ubicación actual de la escultura se ha conseguido inmortalizar en la memoria de los valencianos a este santo y su historia de martirio y muerte, alelegirlo por su proximidad al muladar, lugar que según la tradición fue arrojado su cuerpo. Tras la conquista cristiana de la ciudad el rey Jaume I decidió construir una ermita de planta cuadrada para conmemorar al santo mártir a imitación de las que se hacían en Occidente para recuperar la tradición del protomártir (Panach, 2017). A lo largo de los siglos ha ido sufriendo diversas remodelaciones, pero a mediados del siglo XX se decidió construir una de nueva planta dejando como vestigios la cripta y restos de muro.

Francesc de Vinatea [4]: escultura en bronce y pedestal de piedra realizada por el escultor Manuel Rodríguez Vázquez y cuya inauguración tuvo lugar en 1993, se encuentra en la plaza del Ayuntamiento.

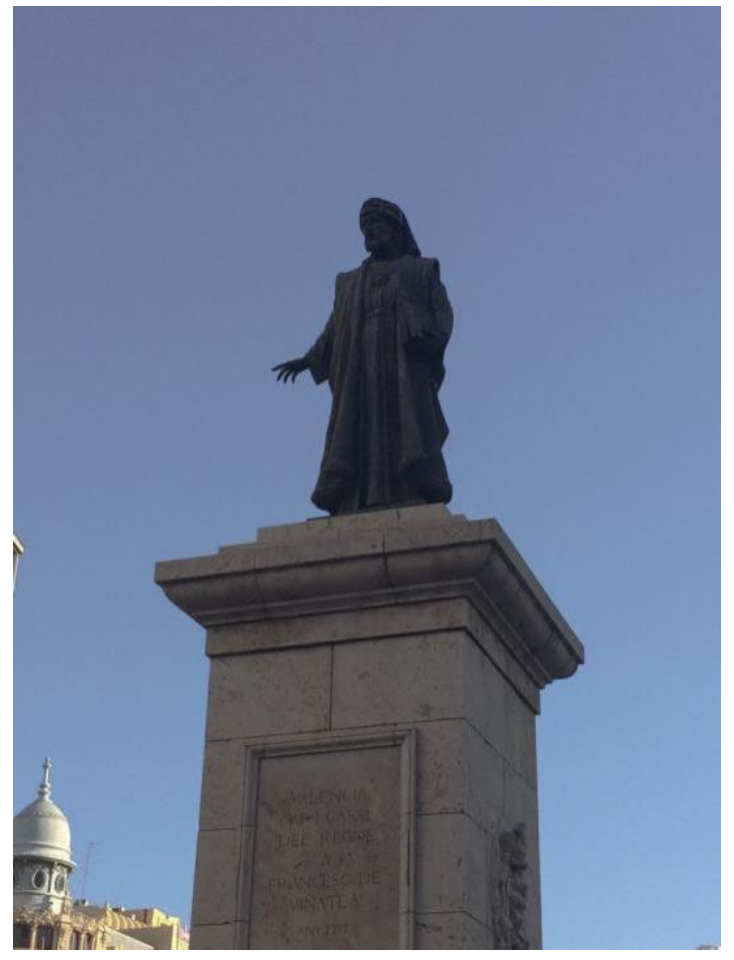

4. Francesc de Vinatea

Francesc de Vinatea, elevado a la categoría de héroe por la historiografía romántica, “ocupa una importante posición en la imaginación colectiva de la sociedad valenciana"9, convirtiéndose en defensor dels furs ${ }^{10}$. Realmente actuó como parlamentario en 1330 alzando su voz ante el peligro que suponía la enajenación del patrimonio real por parte del rey de Aragón Alfons el Benigne (Roca

\footnotetext{
${ }^{9}$ BAYDAL SALA, Vicent (2011), “A figura de Francesc de Vinatea no Reino de Valência. Da crónica real aos documentos archivísticos (1331-1332)”, Mirabilia: Revista Electrônica de História Antiga e Medieval, pp. 214-237

${ }^{10}$ Els Furs, en castellano Los Fueros del Reino de Valencia, fueron las leyes que el rey Jaime I juró y promulgó en 1261 y que rigieron el Reino de Valencia hasta 1707, año en que se abolieron.
} 
Traver, 2003). A este respecto en el mismo pedestal, en la parte posterior, encontramos una inscripción que destaca la labor y actuación de Francesc de Vinatea durante la problemática decisión real $^{11}$. El espacio que acoge la figura de Francesc de Vinatea es la Plaza del Ayuntamiento, lugar donde se encuentra la Casa Consistorial de Valencia, sede del gobierno local y que se ocupa de su administración. La relación que se establece entre el espacio urbano y la figura del "héroe” se subraya por su identificación como defensor del parlamentarismo como jurado (Cardells y Gil,2016).

\section{Conclusiones}

La lectura e interpretación del vínculo entre esculturas públicas y ubicación urbana nos ha llevado a las siguientes conclusiones:

Revalorizar la localización enraizada de las esculturas públicas en el ámbito urbano a partir del papel de cuatro ejemplos de estatuaria coherente con su espacio en la ciudad de Valencia, destacando la figura y su lugar correspondiente en el imaginario colectivo de los valencianos.

Cartografiar el espacio urbano desde los rasgos de las esculturas mostradas: zona eclesiástica, centro político, modernidad del ensanche y nervio espiritual y de tráfico comercial.

Establecer un marco histórico-artístico como hilo conductor de la explicación de la historia de Valencia, como punto de partida para una educación en la sensibilidad artística, destacando la labor del arte como generador de contenidos y contenedor de ideas.

Destacar la importancia y comprensión de la historia local como parte integrante de una historia regional y nacional, entendido como un proceso racional que parte de lo concreto hacia lo general. Asimismo, esto nos lleva a comprender la historia como un trabajo de interpretación y análisis marcado por la interdisciplinariedad y la complementariedad.

\section{Referencias bibliográficas}

ALMELA i VIVES, F. (1989). El Marqués de Campo. Capdavanter de la burguesía valenciana (1814-1889). Ajuntament de València: Valencia

\footnotetext{
11 “FRANCESC VINATEA / JURAT DE LA CIUTAT / IMPEDI EN 1333 A / ALFONSO II EL CONTRAFUR / QUE DIVIDIA EL REGNE / DE VALENCIA A FAVOR / DE L'INFANT FERRANDO / FILL DE / LEONOR DE CASTELLA / "REINA, REINA DEL NOSTRE / POBLE ES FRANC E NO ES / AIXI SUBJUGAT CON ES LO / POBLE DE CASTELLA CAR / ELLS TENEN A NOS COM / A SENYOR E NOS A ELLS / COM A BOONS VASALLS / E COMPANYONS / ALFONS II BENIGNE / REI DE VALENCIA”
} 
APARICI NAVARRO, Miguel (2005). Guía del Convento de Santo Domingo, Antigua Capitanía General y sede actual de la Fuerza de Maniobra. Bancaja: Valencia

BAYDAL SALA, Vicent (2011), “A figura de Francesc de Vinatea no Reino de Valência. Da crónica real aos documentos archivísticos (1331-1332), Mirabilia: Revista Electrônica de História Antiga e Medieval, pp. 214-237

CARDELLS MARTÍ, Francisco Abelardo (2014), “La Valencia bajomedieval y su integración espacial: percepción del área, dimensión comunitaria y representación simbólica del término", en Mundos medievales. Espacios, sociedades y poder, Vol II, pp. 1137-1148

CARDELLS MARTÍ, Francisco Abelardo y GIL SABIO, Noelia (2016), "Referentes históricos en la escultura pública", en CHAVES MARTÍN, Miguel -Ángel (Ed.) (2016). Ciudad y artes visuales, Grupo de Investigación Arte, Arquitectura y Comunicación en la Ciudad Contemporánea, Universidad Complutense: Madrid, pp. 151-160

CORBÍN FERRER, Juan-Luis (1996). El ensanche noble de Valencia: entre Colón y Gran Vía Marqués del Turia. Federico Domenech: Valencia

DE LAS HERAS ESTEBAN, Elena (2003). La escultura pública en Valencia. Estudio y catálogo. Universitat de València, Servei de publicacions: Valencia

DE LECEA, Ignasi (2006). “Esculturas y espacio público en la ciudad de Barcelona”, On the w@terfront, n 8, pp. 13 29. Recuperado de http://www.raco.cat/index.php/Waterfront/article/viewFile/217153/293972 (Consulta: 30/11/2017).

FORCADA COMINS, Vicente y DE GARGANTA, José M. (Dir.) (1956). Biografía y escritos de Sant Vicent Ferrer. Editorial Católica: Madrid

GIL SALINAS, Rafael (2003), “La escultura pública en Valencia y los artistas valencianos”, en LACARRA DUCAY, Carmen y GIMÉNEZ NAVARRO, Cristina (coord.), Historia y política a través de la escultura pública 18201920. Institución Fernando el Católico: Zaragoza, pp. 63-88

MARTINEZ RODA, Federico (1998). Valencia y las Valencias: su historia contemporánea (1800-1975). Universidad San Pablo CEU: Valencia

MERLO FUERTES, José Luis (2014), “Luis Albert y los primeros planes urbanísticos de la Diputación de Valencia”, VCL arquitectura, volumen 1(2), pp. 63-88

MESTRE MARTÍ, María (2011). Viena en la arquitectura modernista de Valencia. Influencias del Jugendstil Vienés en el Modernismo de Valencia. Editorial Universitat Politècnica de València: Valencia

MONTOLIU SOLER, Violeta (2002). Monumentos conmemorativos de Valencia, memoria esculpida de una ciudad: 1875-1936. Real Academia de Cultura Valenciana: Valencia

MORANT, Ana María (2015), "Un coleccionista desconocido en la corte de Alfonso XII: el Marqués de Campo”, Matèria, $\mathrm{n}^{\circ} 9$, pp. $115-127$

NAVARRO SORNI, Miguel (2016), “San Vicente Mártir en su contexto histórico y en las homilías de San Agustín desde una hermenéutica Fe Razón", Fides et Ratio n 1, pp. 131-146 
PALOMERO GONZÁLEZ, José Antonio y ALVARIÑO SERRA, Patricia (2016), "La importancia de higienismo y la potabilización del agua en la ciudad de Valencia”, Investigaciones Geográficas, nº 65, enero-junio, pp. 45-55

PANACH, Víctor y CEBRIÁN, J. (2017), “La configuración de la Iglesia Valentina desde el martirio de san Vicente (304) hasta el Concilio Valentino (546) en tiempos de Justiniano", en CARDELLS, F. A. (Coord). Espiritualidad y territorio. UCV: Valencia, pp. 17-28

REVISTA DE ESCULTURA PÚBLICA ESCULTURA URBANA. Recuperado de http://www.esculturaurbana.com (Consulta:17/11/2017). ISSN 1988-5954

ROCA TRAVER, Francisco (2003). Alfonso II el Benigno, rey de Valencia. Vol. 6 de Biografías Valencianas, Diputación de Valencia: Valencia

TABERNER PASTOR, Francisco (2013), “Arquitectura y desarrollo urbano”, en Notas sobre el Ensanche de valencia de 1884, n 13 de Arte Valenciano, Volumen XCIV, pp. 181-200

VICIANO NAVARRO, Pau (1993), “Entre l'erudició i e pamflet polític: la figura de Francesc Vinatea en la societat valenciana", Revista d'història medieval, 4, pp. 263-274.

ZARAGOZÁ CATALÁN, Arturo (1995), “Antiguo Convento de Santo Domingo”. Direcció General de Patrimoni Artístic: Valencia en ZARAGOZÁ CATALÁN, Arturo (1995), Valencia, Arquitectura Religiosa. Tomo X, en (1995) Monumentos de la Comunidad Valenciana. Direcció General de Patrimoni Artístic: Valencia 